\title{
Primary bone lymphoma of the humerus (about a case and literature review)
}

\author{
Mounir Yahyaoui*, Soufiane Aharram, Jawad Amghar, Omar Agoumi and Abdelkrim Daoudi \\ Orthopedic and trauma surgery A department, university hospital Mohammed 6th, Oujda Morocco
}

\begin{abstract}
Primary localised bone lymphoma is a rare affection prevalent in humans. It often involves long bones, but can be found in all the skeleton bones. The diagnosis is based on the anatomopathological study with immunohistochemical study of a bone biopsy. Treatment is based on chemotherapy (with or without anti-CD20) followed by radiotherapy. The prognosis of primary localised bone lymphoma is very favorable. We also report the first diagnosed case of primary bone lymphoma of the humerus since the inauguration of our university hospital (since September 2014).
\end{abstract}

\section{Introduction}

The World Health Organization (WHO) defined primary bone lymphoma in 2002 as a single bone lesion with or without regional lymph node involvement or multiple bone injuries without distant visceral or lymph node involvement [1]. It is a rare entity, in fact primary bone lymphomas represent 1-3\% of no Hodgkin's lymphomas, $5 \%$ of extra-ganglionic no Hodgkin's lymphomas and 3\% of primary bone tumors $[2,3]$.

\section{Clinical case}

H.Y, 77 years old, female, hypertensive, no insulinodependant diabetes, in good condition, who had been complaining for 1 month of mixed pains in his right arm without functional impotence or other associated signs. The musculoskeletal examination did not reveal any mass in the arm or inflammatory signs, apart from a slight pain on palpation. The examination of the locoregional lymph node areas was without particularities, also the general somatic examination.

Standard radiographs of his right humerus showed a small lytic lesion of the external cortex of the humeral shaft without a periosteal reaction (Figure 1). The TAP CT revealed the same lesion with no soft tissue extension (Figure 2), no suspicious lesions on the thoracoabdomino-pelvic views, and no lymphadenopathy. The biological assessment did not reveal any anomalies, in particular a $\mathrm{LDH}$ concentration in the normal fork.

The patient underwent a bone biopsy, the immunohistochemical result of which was in favor of diffuse large B cell lymphoma (Figure 3). After a multidisciplinary concertation meeting, the patient is a candidate for 6 cures of CHOP chemotherapy combined with anti$\mathrm{CD} 20$, and radiotherapy thereafter.

\section{Discussion}

Primary bone lymphomas are defined by the primary location of a malignant lymphoma in the bone, with a negative extension assessment within 6 months of the positive diagnosis. Another inclusion criterion is unique bone localization, but some authors integrate multifocal

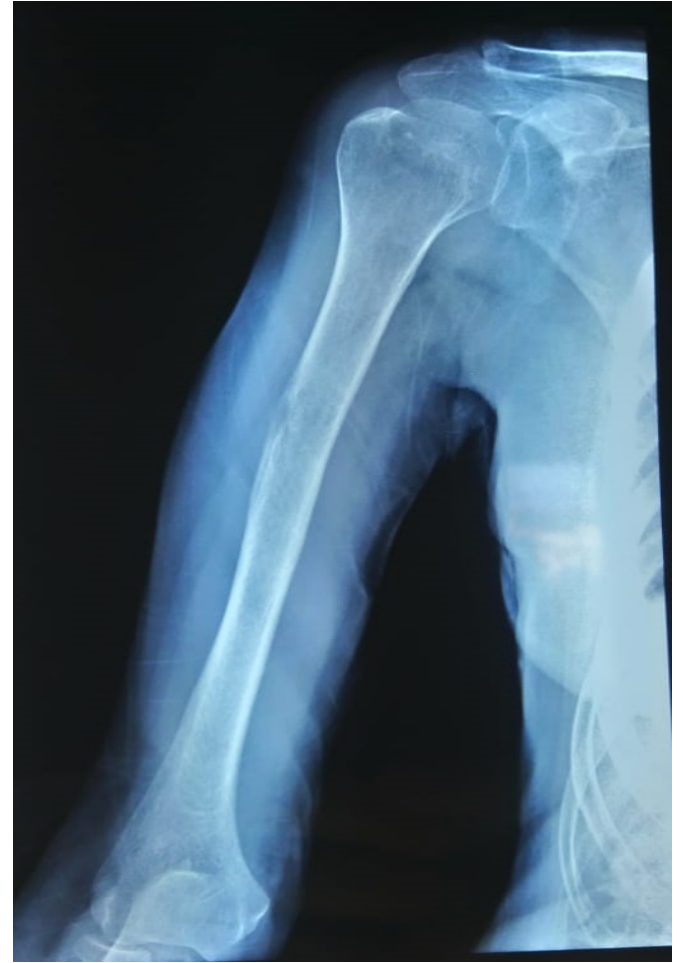

Figure 1. Front radiograph of the right humerus (osteolytic lesion of the external cortex of the right humeral shaft, without periosteal reaction)

${ }^{\star}$ Correspondence to: Mounir Yahyaoui, Orthopedic and trauma surgery A department, university hospital Mohammed 6th, Oujda, Morocco, E-mail: mounirdeloin@live.fr

Key words: primary bone lymphoma, chemotherapy, radiotherapy, biopsy

Received: April 12, 2020; Accepted: April 22, 2020; Published: April 27, 2020 


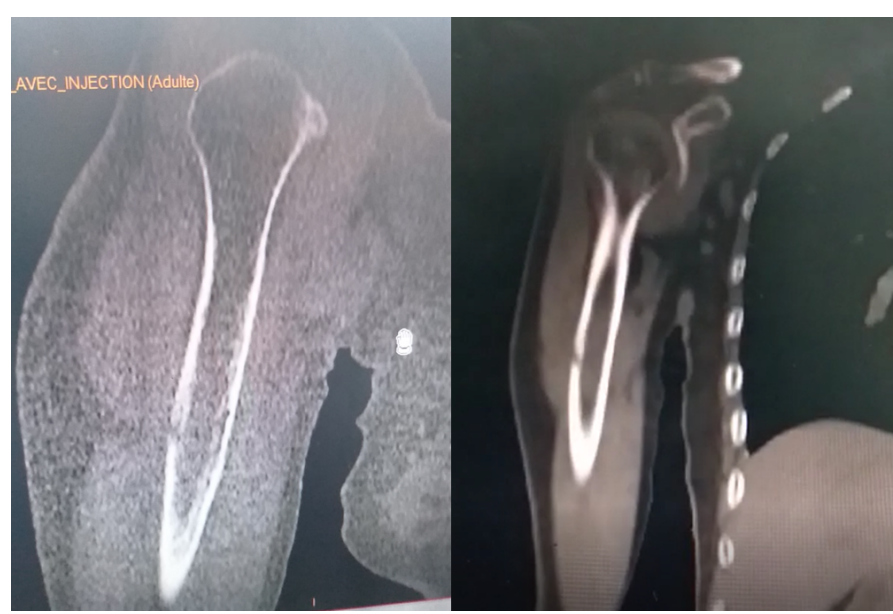

Figure 2. CT scans of the right arm in bony and parenchymal views in the context of TAP CT (no soft tissue extension, no other suspicious lesions or lymphadenopathy)

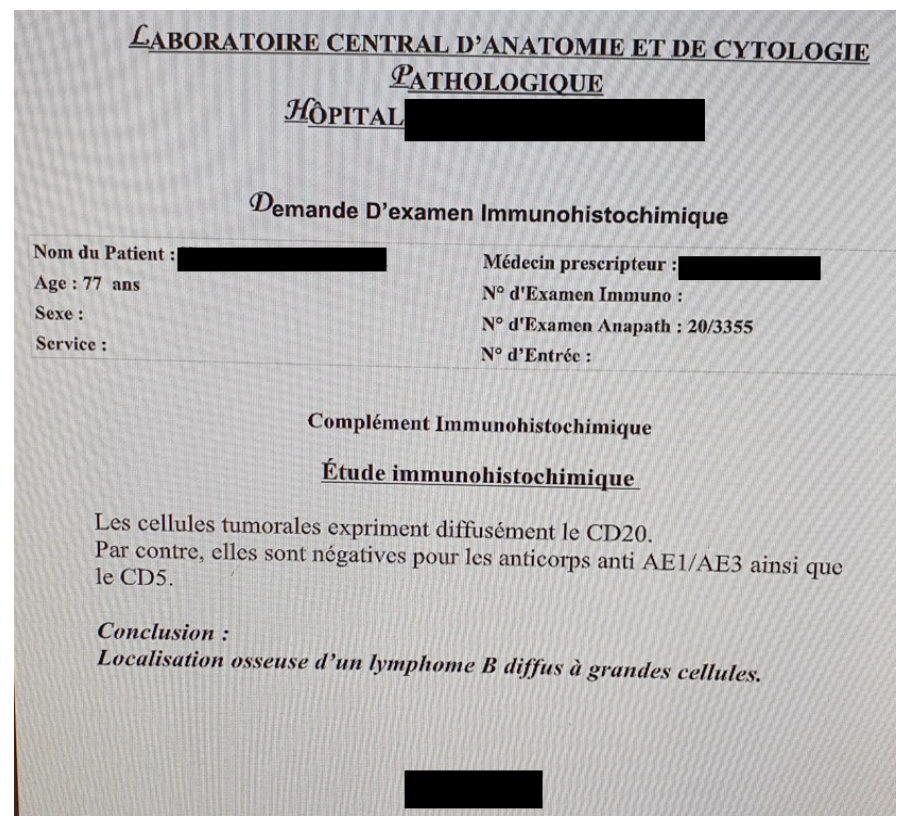

Figure 3. Immunohistochemistry of bone biopsy (diffuse large B cell lymphoma)

bone lymphomas into primary bone lymphomas [2]. Primary bone lymphomas are rare. They predominate in men of median age between $40-60$ years $[4,5]$. Primary bone lymphoma is at a localized stage if it is classified stage IE or IIE with no symptom B and without tumor mass greater than or equal to $10 \mathrm{~cm}$ (bulky). It is advanced if it is stage III, IV, IE or IIE with B symptoms or bulky [6].

The most frequent anatomical sites are bones with significant hematopoietic activity (long bones, vertebrae, pelvis, ...) [7]. Mandibular involvement represents $2-17 \%$ of published cases [8], the clavicle is reached in $4 \%$ of cases [8]. Bone pain is the most frequently reported symptom (60-100\%). Local swelling is found only in $50 \%$ of cases [6]. A pathological fracture rarely reveals the disease (10-17\% of cases) [1]. Spinal cord compression is a symptom revealing of certain vertebral localizations [1,8]. B symptoms are rarely seen [4].

The radiological appearance of primary bone lymphoma is variable and no specific. It is often a destructive osteolytic lesion arising from the bone marrow. The lesion can be focal and well limited or cause a cortical rupture, a pathological fracture, or even extend to the soft tissue [4]. Bone scintigraphy often shows hyperfixation of the tracer which is not specific [4]. MRI is more suitable for tumor evaluation. It typically shows a bone marrow replacement with a T1 hyposignal and a T2 hypersignal. MRI also makes it possible to study the extension to the soft tissue and to the spinal cord and to look for signs of compression [4].

The diagnosis is based on a bone biopsy with an anatomopathological and immunohistochemical studies [6]. Primary bone lymphomas are no Hodgkin large B cell lymphomas in $80 \%$ of cases $[3,4]$. They are often localized [3]. Radiotherapy was the standard curative treatment for primary bone lymphoma localized before the therapeutic combination area. Currently, chemotherapy followed by radiotherapy has demonstrated its interest in overall survival without relapse, and constitutes the reference treatment regimen [6,9]. CHOP chemotherapy (cyclophosphamide, doxorubicin, vincristine, and prednisone) is the standard first line treatment. The recommended number of cures is 3-8 [8,9]. There is likely to be no benefit from using more intensive chemotherapy such as ACVBP (doxorubicin, cyclophosphamide, bleomycin, vincristine and prednisone) $[5,10]$. The literature data concerning the addition of anti-CD20 to chemotherapy are discordant $[8,9]$. In case of response, consolidation radiotherapy is delivered in the initial tumor volume with a margin of $2 \mathrm{~cm}$. A dose of 40-50 Gy in standard spreading and fractionation is recommended $[4,6,8]$. In rare cases of no response to chemotherapy, there is no clear attitude in the literature.

Currently, the place of surgery in the management of primary bone lymphomas is limited. The initial surgical biopsy allows the diagnosis. The role of surgery comes down to the treatment of pathological fractures and threatening lesions of fracture or spinal instability [4]. In case of spinal cord compression, a laminectomy is often decided to free the marrow and avoid the installation of an irreversible neurological deficit [11].

The prognosis of localized primary bone lymphomas is excellent. The survival probability at 5 years is at stage I of $90 \%$ [8]. The IPI or international prognostic index (including age, general condition, stage, LDH concentration and number of lymph node areas affected) is an independent prognostic factor in terms of overall survival of patients with primary bone lymphoma $[1,8]$ (Table 1$)$.

\section{Conclusion}

Primary localized bone lymphoma is a rare disorder prevalent in humans. It often involves long bones, but can be found in all the skeleton bones. The clinical presentation is not specific. The diagnosis is based on the anatomopathological and immunohistochemical studies of a bone biopsy. Treatment is based on chemotherapy (with or without anti-CD20) followed by radiotherapy. The prognosis for primary localized bone lymphoma is very favorable.

Table 1. The classification used for primary bone lymphoma is that of Ann Arbor, which distinguishes four stages [6]

\begin{tabular}{|c|c|}
\hline Ann arbor stage & Definition \\
\hline IE & Single bone lesion \\
\hline IIE & Single bone lesion with contiguous lymph node involvement \\
\hline III & Bone injury with distant lymph node involvement \\
\hline IV & Multiple bone lesions or a diffuse bone lesion of a long bone \\
\hline
\end{tabular}




\section{Conflicts of interest}

The authors declare that they have no conflicts of interest in connection with this article.

\section{Author contributions}

All the authors contributed to the conduct of this research work and have read and approved the final version of the manuscript.

\section{References}

1. Fletcher CDM, Unni KK, Mertens F (2002) World Health Organisation classification of tumors : pathology and genetics of tumors of soft tissue and bone. Lyon, France : IARC Press p. 299-301.

2. Sans N, Despeyroux-Ewers ML, Loustau O, Vial J, Gandors H, et al. (2004) Imagerie des lymphomes osseux. JFR 2004. Paris : Société française de radiologie.

3. Jawad MU, Schneiderbauer MM, Min ES, Cheung MC, Koniaris LG (2010) Primary lymphoma of bone in adult patients. Cancer 116: 871-9. [Crossref]

4. Mikhaeel NG (2012) Primary bone lymphoma. Clin Oncol 24: 366-370.
5. Beal K, Allen L, Yahalom J (2006) Primary bone lymphoma: treatment results and prognostic factors with long-term follow-up of 82 patients. Cancer 106: 2652-2656.

6. Christie D, Dear K, Stat M, Le T, Barton M, et al. (2011) Limited chemotherapy and shrinking field radiotherapy for osteomlymphoma (primary bone lymphoma): results from the Trans-Tasman Radiation Oncology Group 99.04 and Australasien Leukemia and Lymphoma Group LY 02 prospective trial. Int J Radiat Oncol Biol Phys 80: 1164-1170.

7. Ramadan KM, Shenkier T, Sehn LH, Gascoyne RD, Connors JM (2007) A clinicopathological retrospective study of 131 patients with primary bone lymphoma $:$ a population-based study of successively treated cohorts from the British Columbia Cancer Agency. Ann Oncol 18: 129-135.

8. Dubey P, Ha CS, Besa PC, Fuller L, Cabanillas F, et al. (1997) Localised primary malignant lymphoma of bone. Int J Radiat Oncol Biol Phys 37: 1087-1093.

9. Cai L, Stauder MC, Zhang YJ, Poortmans P, Li YX, et al. (2012) Early-stage primary bone lymphoma: a retrospective, multicenter Rare Cancer Network (RCN) Study. Int $J$ Radiat Oncol Biol Phys 83: 284-291.

10. Reyes F, Lepage E, Ganem G, Molina TJ, Brice P, et al. (2005) ACVBP versus CHOP plus radiotherapy for localised aggressive lymphoma. $N$ Engl J Med 352: 1250-1252.

11. Slimani KA, Belbaraka R, Allam W, Ichou M, Errihani H (2011) Les lymphomes osseux primitifs au Maroc. Étude rétrospective à propos de 12 cas. J Afr Cancer 3: 49-51.

Copyright: (C2020 Yahyaoui M. This is an open-access article distributed under the terms of the Creative Commons Attribution License, which permits unrestricted use, distribution, and reproduction in any medium, provided the original author and source are credited. 